\title{
STATISTICS OF QUASAR LENSING \\ CAUSED BY CLUSTERS OF GALAXIES
}

\author{
K. TOMITA \\ Yukawa Institute for Theoretical Physics, \\ Kyoto University, Kyoto 606-01, Japan
}

\section{Introduction}

Cosmological lens statistics was studied first by Turner, Ostriker, \& Gott (1984), and developed by Hinshaw \& Krauss (1987), Gott, Park, \& Lee (1989), Turner (1990), Krauss \& White (1992), and Wallington \& Narayan (1993). The expected number of lensed quasars in general quasar catalogs was examined by Fukugita \& Turner (1991), Kochanek (1991), and Fukugita et al. (1992), and the number expected in the HST Snapshot Survey (Bahcall et al. 1992, Maoz et al. 1992, 1993) was examined by $\mathrm{Maoz}$ \& Rix (1939) and Kochanek (1993). In most of these studies, galaxies were assumed to be the lenses, and clusters of galaxies played only auxiliary roles. As a result, the expected number of lensed quasars with separations larger than 3 arcsec is negligibly small compared to the total number of lenses. However, about $33 \%$ of observed lensed quasars have image separations larger than 3 arcsec (Maoz et al. 1993, Refsdal \& Surdej 1994). This observational fact is very important.

Clusters of galaxies as lenses, on the other hand, produce conspicuous lensed images like arcs and arclets, whose sources are background galaxies. It is plausible that clusters of galaxies can similarly produce observable lensed images of quasars. In a recent paper (Tomita 1995a) we studied the statistics of lenses due to clusters of galaxies, where we assumed the clusters have a uniform spatial distribution for $z \leq z_{f}(=0.5-0.7)$. In the next section we show the results for various cosmological models, including the super-horizon-scale inhomogeneous model introduced by (Tomita 1995b). 


\section{Lensing due to clusters of galaxies}

For the mass distribution of clusters of galaxies we assumed the mass function derived by Bahcall \& Cen (1993), which is similar in form to the Schechter function for galaxies. The bending angle was derived by regarding clusters of galaxies as isothermal gas spheres with finite cores. The parameterized dependence of the core radius and the velocity dispersion on the mass was normalized using the mean values for X-ray clusters with lensed arc images (e.g. Tyson et al. 1990, Mellier et al. 1993, 1994, Petrosian \& Lynds 1992).

We derived the expected number of lenses in the HST Snapshot Survey taking into account the angular selection function and using the magnification bias model of Maoz \& Rix (1993), and compared the results to the observed sample (Maoz et al. 1993). A limited number of cosmological models could satisfy the following two conditions at the same time: (1) the condition that the expected number of lensed quasars with separation angles larger than 3 arcsec is about $33 \%$ of the total number of lenses, and (2) the condition that the expected total number of lenses equals the observed number. We examined the Einstein-de Sitter model, an open lowdensity homogeneous model with $\Omega_{0}=0.1$ and $\lambda_{0}=0$, a flat homogeneous model with $\Omega_{0}=0.1$ and $\lambda_{0}=0.9$, and the super-horizon-scale inhomogeneous models with $\Omega_{0}=0.1$ or 0.2 in the inner homogeneous region. Of these models, only the flat model with a cosmological constant and the super-horizon-scale model with the low-density inner region satisfied the constraints. This conclusion differs significantly from earlier studies where the only lenses were galaxies formed at epochs $z>3$.

\section{References}

Bahcall, N.A., \& Cen, R., 1993, ApJL, 407, L49

Maoz, D., Bahcall, J. N., Schneider, D. P., Bahcall, N. A., Djorgovski, S., Doxsey, R., Gould, A., Kirhakos, S., Meylan, G., \& Yanny, B., 1993, ApJ, 409, 28

Maoz, D., \& Rix, H.-W., 1993, ApJ, 416, 425

Mellier, Y., Fort, B., \& Kneib, J.-P., 1993, ApJ, 407, 33

Mellier, Y., Fort, B., Bonnet, H., \& Kneib, J.-P., 1994, in Cosmological Aspects of X-Ray Clusters of Galaxies, ed. W.C. Seitter (Dordrecht: Kluwer), 219

Petrosian, V., \& Lynds, R., 1992, in Gravitational Lenses, ed. R. Kayser, T. Schramm, \& L. Nieser (Berlin: Springer-Verlag), 303

Refdal, S. \& Surdej, J., 1994, Rep Prog Phys, 56, 117

Tomita, K., 1995a, PASJ, in press (preprint YITP/U-95-09)

Tomita, K., 1995b, ApJ, 450, 1

Turner, E.L., Ostriker, J.P., \& Gott, J.R., 1984, ApJ, 284, 1

Tyson, J.A., Valdes, F., \& Wenk, R.A., 1990, ApJL, 249, L1 